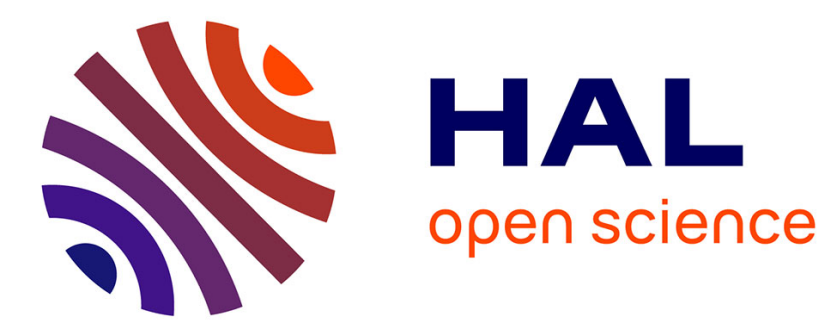

\title{
La topographie cultuelle chrétienne de la région de Potamia-Agios Sozoménos
}

Catherine Vanderheyde

\section{To cite this version:}

Catherine Vanderheyde. La topographie cultuelle chrétienne de la région de Potamia-Agios Sozoménos. Cahiers du Centre d'Etudes Chypriotes, 2004, 34, pp.251-266. halshs-00105124

\section{HAL Id: halshs-00105124 \\ https://shs.hal.science/halshs-00105124}

Submitted on 10 Oct 2006

HAL is a multi-disciplinary open access archive for the deposit and dissemination of scientific research documents, whether they are published or not. The documents may come from teaching and research institutions in France or abroad, or from public or private research centers.
L'archive ouverte pluridisciplinaire HAL, est destinée au dépôt et à la diffusion de documents scientifiques de niveau recherche, publiés ou non, émanant des établissements d'enseignement et de recherche français ou étrangers, des laboratoires publics ou privés. 
Article paru dans « Cahier du Centre d'Etudes Chypriotes 34 »

2004 


\title{
LA TOPOGRAPHIE CULTUELLE CHRÉTIENNE DE LA RÉGION DE POTAMIA-AgIOS SOZOMÉNOS
}

\author{
Andreas NICOLAIDÈS et Catherine VANDERHEYDE
}

\begin{abstract}
Several churches, well preserved or in ruins, are still to be seen in the Potamia-Agios Sozomenos area, as well as in its extension to the neighbouring village of Idalion. The corpus comprises the hermitage of Agios Sozomenos, obviously the more ancient Christian monument of the area, the Byzantine church of Panagia (also called Agios Georgios), the church of the Timios Stavros, in the cemetery of the Potamia village, the ruined or totally destroyed churches of Agia Marina, Santa Caterina and Archangelos respectively, the church of Agios Demetrianos at Agridi, the cemetery church of Agios Mamas at Idalion and Agios Mamas at Agios Sozomenos, the only known Cypriot example of a Gothic church in a rural area. It is accurate that there are numerous Byzantine or Gothic monuments which provide clues about the history of the valley in relationship with its water supply.
\end{abstract}

Si beaucoup de temps a passé depuis la construction ou l'aménagement des lieux de culte de la région de Potamia (Fig. 1), la mémoire des saints auxquels ils sont dédiés est encore bien vivace chez les habitants. Lorsque l'on consulte l'étude anthropologique menée à Potamia par B. Karatsioli en 2001, on est en effet surpris que les habitants attribuent encore actuellement une notion de protection aux saints dont les « demeures » entourent leur village. La renommée de ces saints protecteurs, mais aussi guérisseurs, dépasse même les frontières du village de Potamia, puisque les femmes du village voisin de Lympia participaient à une sorte de pèlerinage : leur itinéraire débutait à Hagia Mavri ${ }^{1}$, passait ensuite par Hagia Marina, puis Santa Caterina et se terminait à la chapelle de l'Archange ${ }^{2}$. Si elles exercent encore une certaine force d'attraction cultuelle, ces constructions sont aujourd'hui les plus endommagées de la région étudiée. D'autres lieux de culte sont mentionnés dans la recherche anthropologique ; on peut par exemple y lire ceci : «le village de Potamia n'avait jamais de problèmes de saints car il

1. Ce lieu de culte n'a pas pu être identifié à cause de sa situation dans un champ de mines. D'après l'étude anthropologique basée sur les témoignages des habitants de Potamia, menée par B. Karatsioli en 2001, on sait que ce lieu de culte était important pour les habitants de Potamia et que des gens pouvaient y être enterrés, $c f$. Karatsioli 2001, p. 23.

2. Ibid., p. 40-41. 
était protégé tout autour de saints: Hagia Marina, Hagia Mavri, Santa Katerina, Panagia de Agios Sozoménos, Agios Démétrianos, Pallourkotissa... " ${ }^{3}$. Parmi ces saints protecteurs liés au village de Potamia et à ses environs immédiats, la Vierge occupe naturellement une place privilégiée dont témoigne notamment la célébration de la fête de sa Présentation au temple, le 21 novembre, qui donne lieu à une «panigiris » à l'ermitage de la Vierge de saint Sozomène ${ }^{4}$.

Ces quelques informations tirées de l'étude anthropologique indiquent déjà que l'étude de la géographie sacrée de la région de Potamia est susceptible de fournir des renseignements sur les spécificités de ces lieux de culte et sur les rapports qui s'établissent entre eux. Par ailleurs, l'étude de leur architecture, de leur décor et de leur ordre de succession dans le temps permet aussi de mieux comprendre l'histoire du peuplement du territoire envisagé sur une longue durée 5 .

Par ailleurs, certains de ces lieux de culte, tels que l'ermitage, la chapelle d'Agia Mavri et celle de l'Archange, ainsi que la Pallourkotissa, étaient de toute évidence liés à des points d'eau : un puits et un culte de l'eau sont attestés à l'ermitage, une source est associée à Agia Mavri ; la chapelle de l'Archange est construite près du cours du Yialias; Saint-Démétrianos est proche d'un moulin et d'un grand réservoir d'eau ; et la Pallourkotissa est située à proximité d'une noria. L'implantation de ces lieux de culte est donc relative à l'alimentation en eau qui était essentielle au développement des cultures et plus largement à l'autosubsistance de la région.

\section{Agios Sozoménos et ses environs}

\section{L'ermitage de saint Sozomène (Fig. 2)}

Tenter de reconstituer l'histoire de Sozomène est une tâche ardue, car beaucoup de zones d'ombre subsistent sur l'origine de ce personnage et la période à laquelle il a vécu. La mention de ce saint dans la chronique de Léontios Machairas (milieu du XVe siècle) nous informe qu'il faisait partie des trois-cents saints issus de familles de croisés établis en Terre-Sainte, arrivés à Chypre après avoir fui la Palestine à la suite de la prise de Jérusalem par les Musulmans en 1187, et consécutivement à la chute de Saint-Jean d'Acre en $1291^{6}$. Cependant, le récit qui concerne ces 300 saints "allemands » de Chypre présente un caractère légendaire évident et il semble probable que les chroniqueurs des $X V^{\mathrm{e}}$ et $\mathrm{XVI} \mathrm{I}^{\mathrm{e}}$ siècles, tels Léontios Machairas ou Étienne de Lusignan ${ }^{7}$, aient associé des faits appartenant à des époques différentes sans s'efforcer de

3. Ibid., p. 16.

4. Karatsioli 2001, p. 41.

5. L'étude approfondie de chacun de ces lieux de culte fera partie de l'étude d'ensemble sur le territoire de Potamia-Agios Sozoménos, coordonnée par N. Lécuyer.

6. Dawkins 1932, vol. 1, § 32, p. 30-31; § 77, p. 70-71.

7. Étienne de Lusignan, Description de toute l'isle de Chypre, Paris, 1580, f. 83. Voir également Kyprianos 1902, p. 524-525, et Makarios III 1968, p. 40, nº 215. 
les ordonner chronologiquement ${ }^{8}$. Comme l'a bien montré C.P. Kyrris, ce récit compile en effet un nombre important et hétéroclite de données sur des saints martyrs dont l'existence n'est pas toujours assurée ${ }^{9}$. Il est donc possible que saint Sozomène de Potamia ait vécu avant l'arrivée de ces saints qui menèrent une vie ascétique à Chypre au XIII' siècle, et que l'excavation de son ermitage soit consécutive aux incursions des Sarrasins qui ont éprouvé l'île entre 690 et 697. Étant donné la similitude entre la destinée de l'ermite Sozomène et celle des ascètes du XIII ${ }^{\mathrm{e}}$ siècle venus à Chypre en quête de solitude, il semble qu'une sorte de "glissement » ait été opéré dans les chroniques des $\mathrm{XV}^{\mathrm{e}}$ et $\mathrm{XVI} \mathrm{I}^{\mathrm{e}}$ siècles, ce qui expliquerait la mention de Sozomène parmi les « 300 saints allemands».

L'étude de l'ermitage rupestre de saint Sozomène et de son décor peint laisse supposer qu'il y mena une vie ascétique, et qu'il y fut peut-être enseveli. L'agencement d'une fosse rectangulaire semble avoir été conçu dès le départ pour recevoir la sépulture du saint ${ }^{10}$. Par ailleurs, les fresques ornant l'ermitage constituent une source d'information primordiale sur la vie et les miracles de Sozomène. La première couche des peintures a été datée par A. Papageorgiou du $\mathrm{X}^{\mathrm{e}}$ siècle sur des critères stylistiques ${ }^{11}$, et livrerait donc un terminus ante quem à la réclusion de Sozomène. Cette dernière pourrait être consécutive aux guerres arabo-byzantines, qui sont à l'origine du dépeuplement de certaines régions littorales de Chypre dès le VII ${ }^{e}$ siècle. L'unique représentation issue de cette première phase de peintures, actuellement conservée au musée d'Art byzantin-Fondation Makarios III à Nicosie, montre six saints vêtus de l'habit monastique : ce sont Sozomène, Jason, Galaktiôn, Agapios, un moine non identifié et Aristôn. A. Papageorgiou date la seconde couche de fresques couvrant les parois de l'ermitage du saint de la période paléologue, sur des critères stylistiques également ${ }^{12}$. Ce cycle détaillé de peintures de belle facture représente divers épisodes de la vie et des miracles du saint, et il suggère peut-être un développement de son culte dans la région de Potamia au XIVe siècle. L'événement suivant, rapporté par Léontios Machairas, laisse par ailleurs supposer la renommée et l'efficacité des reliques de ce saint à cette même période. Lorsqu'il se rendit à Chypre, vers le milieu du XIVe siècle, le patriarche Ignace II d'Antioche commanda une croix en bois. Cette dernière, portée en

8. Delehaye 1907, p. 251-252.

9. Kyrris 1993, p. 203-235, surtout p. 215.

10. Cette sépulture était peut-être analogue au sarcophage encore conservé dans l'ermitage rupestre de saint Eutychios à Nisou, situé à quelques kilomètres du village d'Agios Sozoménos, cf. Papageorgiou 1999, p. 44.

11. Ibid., p. 48.

12. A. Papageorgiou pense que ces fresques ont été exécutées durant le premier tiers du XIV e siècle. Elles sont en effet stylistiquement très proches de celles qui décorent le narthex de la Panagia Phorviotissa à Asinou, datées de 1333, cf. Papageorgiou 1999, p. 52. 
procession afin de conjurer les calamités des sauterelles et de la sécheresse, contenait les reliques de divers saints, parmi lesquels saint Sozomène de Potamia ${ }^{13}$.

Le fait que la fête de saint Sozomène ait lieu le 21 novembre, et que ce jour coïncide exactement avec la célébration de la Présentation de la Vierge au Temple ${ }^{14}$ pourrait expliquer que le culte marial se soit progressivement associé, puis superposé au culte de saint Sozomène. Actuellement, l'ermitage est d'ailleurs appelé «Panagia d'Agios Sozoménos ", double dédicace insolite qui pérennise bien la trace des deux dévotions. De plus, dans l'imaginaire des habitants de Potamia, la Vierge est fermement associée à l'ermitage de saint Sozomène. En témoignent ces deux passages consignés dans l'étude anthropologique: "À Agios Sozoménos, beaucoup de gens voyaient la Vierge se promener le soir, tenant une bougie dans ses mains; elle se dirigeait vers la grotte et récitait une litanie"; et encore : "Quand Panaghia de Agios Sozoménos est allée habiter dans le village, elle a frappé à trois endroits de la montagne ; le troisième était le bon et elle en a fait son habitat ${ }^{15}$. La ferveur de la dévotion portée à la Vierge de saint Sozomène et l'action bienfaisante de cette dernière en faveur de la fécondité des jeunes femmes mariées sont également attestées ${ }^{16}$.

\section{Saint-Mamas}

Cette église (Fig. 3) constitue l'un des rares exemples d'édifice gothique construit dans la campagne de l'île de Chypre. L'étude métrologique révèle un édifice d'une grande régularité et d'un traçage très simple dont la réalisation - au moins de ce qu'on peut observer actuellement - a été régie par un module très précis de $106,6 \mathrm{~cm}{ }^{17}$. Certains traits architecturaux, tels que les bases des colonnes, leurs chapiteaux et les fines colonnettes portées par ces chapiteaux, rappellent les dispositifs des cathédrales gothiques françaises de Nicosie et de Famagouste. G. Jeffery a par ailleurs observé des similitudes entre certains détails architecturaux de Saint-Mamas et de l'église de l'Archange à Lakatamia ${ }^{18}$.

Néanmoins, la datation de cette église est controversée. C. Enlart estimait qu'elle ne pouvait être antérieure au $\mathrm{XV}^{\mathrm{e}}$ siècle. Il supposait une filiation entre cette église et l'abbaye Saint-Mamas de Morphou, dont la construction se caractérise en effet par un mélange d'influences gothiques et byzantines et aurait eu lieu, toujours selon lui, à la

13. Dawkins 1932, vol. 1, § 77, p. 70-71; Kleridès 1938, p. 110 ; Kyrris 1999, vol. VII, col. 1079-1082.

14. Ibid., col. 1081 .

15. Karatsioli 2001, p. 22-23.

16. Ibid., notamment p. 41.

17. L'étude métrologique de Saint-Mamas est due A. Cheilan, doctorant à l'Université de Provence.

18. Jeffery 1983, p. 206. 
fin du XIVe ou au début du XV $\mathrm{V}^{\mathrm{e}}$ siècle ${ }^{19}$. Ces similitudes architecturales et décoratives expliquent peut-être aussi pourquoi Enlart attribuait à l'église du village d'Agios Sozoménos la dédicace de saint Mamas - martyr originaire de Cappadoce dont le cercueil vint échouer dans la baic de Morphou, selon la légende. Son immense popularité à Chypre a fait de lui un saint national ${ }^{20}$.

Comme l'avait déjà constaté C. Enlart, plusieurs incohérences sont visibles dans la construction et le décor architectural. Si les deux enfeus pratiqués dans le mur sud indiquent la destination funéraire de cette église, il n'en demeure pas moins curieux que leur décor sculpté n'ait jamais été terminé. De plus, les piliers qui flanquent ces enfeus devaient manifestement recevoir la retombée d'un fronton qui n'a jamais été exécuté. Certains chapiteaux du portail ouest de l'église sont épannelés, tandis que d'autres sont achevés. Les chapiteaux et les bases des colonnes adossées aux extrémités est et ouest des collatéraux présentent aussi un décor partiellement exécutés : complets à l'ouest, ils demeurent inachevés à l'est.

À ces incohérences indéniables dans le décor architectural s'ajoutent des problèmes liés au mode de couverture de cette église, qui semble ne jamais avoir été achevée : en témoigne l'absence de voûtes sur les nefs. De plus, la partie supérieure des murs de la nef centrale et des murs latéraux ne paraît pas avoir été terminée. Enfin, on peut difficilement admettre que les fines colonnettes, reposant sur des petits socles débordant de moitié du lit d'attente des chapiteaux des colonnes de la nef centrale, aient été suffisantes pour supporter la retombée du système de voûtes adopté pour l'église.

C. Enlart mettait en rapport l'ensemble de ces éléments inachevés avec un arrêt de la construction de l'église. Si l'hypothèse d'Enlart est en partie convaincante, certains détails architecturaux, tel le décor des enfeus, ne permettent pas d'exclure une réalisation à la fin du $X V^{e} s$., voire au $X V I^{e}$ siècle. Les incohérences observées dans la construction pourraient peut-être s'expliquer par un arrêt des travaux suite à un tremblement de terre. Plusieurs séismes sont en effet signalés à Chypre durant les $X^{\mathrm{e}}$ et $X V I^{\mathrm{e}}$ siècles ${ }^{21}$.

\section{Saint-Georges / la Panagia}

Le vocable de Saint-Georges attribué à cette église (Fig. 4) a probablement été dicté récemment par le fragment de décor peint conservé sur sa façade nord ${ }^{22}$, où l'on peut reconnaître ce saint cavalier avec sa chlamyde rouge flottant au vent derrière lui. D'après certaines informations consignées dans l'étude anthropologique, cette église semble plutôt avoir été consacrée à la Vierge et présente, selon toute vraisemblance, un rapport étroit avec le culte voué à la Vierge dans l'ermitage de saint Sozomène. L'étude

19. Enlart 1899, p. 194-198.

20. Delehaye 1907, p. 272 ; voir également Mouriki 1993, p. 249 -251.

21. Gilles Grivaud a notamment repéré dans les sources écrites douze mentions de séismes pour le XVI $\mathrm{I}^{\mathrm{e}}$ siècle, $c f$. Grivaud 1998, p. 432.

22. Cette fresque fragmentaire se situe à $1,52 \mathrm{~m}$ du sol et se prolonge sur une largeur d'environ 1,23 m. 
anthropologique nous rapporte en effet que la veille de la fête d'Agios Sozoménos, l'espérinos, se déroulait à l'église de la Panagia "du bas ", à savoir l'église qui nous occupe, tandis que lors de la fête de la Vierge, le 21 novembre, l'office était célébré à l'église de la Panagia de saint Sozomène ${ }^{23}$.

L'iconographie des peintures murales situées à l'intérieur de Saint-Georges conforte, s'il en est besoin, le vocable de la Vierge ${ }^{24}$. De fait, sur la paroi sud de la nef, dans le voisinage immédiat de la clôture du bêma, subsiste une icône murale peinte, inscrite dans un encadrement décoratif, représentant la Vierge Hodigitria : il s'agit d'un emplacement traditionnel dévolu au saint dédicataire ${ }^{25}$. En outre, on parvient à identifier sur les autres fragments peints, sur la paroi sud du bêma, saint Zosime présentant une cuillère et un calice liturgiques, qui fait partie de la scène de la Communion de Marie l'Égyptienne qui n'est plus conservée. Subsiste encore un saint diacre (Étienne ?) peint sur le piédroit méridional de l'abside.

Cet édifice est une modeste église à nef unique, que C. Enlart qualifie de «petit et misérable monument byzantin à voûte en berceau " 26 . L'étude du parement extérieur indique clairement une reprise de la construction vers l'ouest. Par ailleurs, deux des trois bacini en céramique engobée et glaçurée de Çanakkale sont encore encastrés au sommet de la voûte précédant le cul de four de l'abside. Ceux-ci permettent de dater la réparation de cette voûte de la fin du XVIII ${ }^{\mathrm{e}}$ ou du début du XIX ${ }^{\mathrm{c}}$ siècle ${ }^{27}$. En revanche, le style de peintures murales fournit une datation des $X I V^{\mathrm{e}}-\mathrm{XV} \mathrm{V}^{\mathrm{e}}$ siècles.

\section{Agia Marina}

Cet édifice en ruine était une chapelle à nef unique située sur les hauteurs de Potamia dominant au loin le village d'Agios Sozoménos. En 1918, G. Jeffery signalait la présence de peintures murales, mais plus aucune trace de celles-ci ne subsiste actuellement ${ }^{28}$. L'étude anthropologique rapporte l'existence «d'un cimetière très ancien » à l'endroit de l'église désormais en ruine, et relaye l'assertion selon laquelle cette dernière «n'appartenait pas aux Maronites, mais aux Latins » ${ }^{29}$. Cette remarque conforte l'hypothèse de G. Jeffery suivant laquelle Agia Marina serait un des paraecclesia du couvent des Franciscains de Pyroï ${ }^{30}$.

23. Karatsioli 2001, p. 11.

24. Le vocable de la Vierge est également cité par Jeffery 1983, p. 206, et par Gunnis 1973, p. 204-205.

25. Voir à ce sujet Nicolaides 1996, p. 104 et suivantes.

26. Enlart 1899, p. 194.

27. Voir à ce sujet François, Vallauri 2001, p. 544, fig. 11.3

28. Jeffery 1983, p. 204.

29. Karatsioli 2001, p. 32. Les informations sont assez contradictoires à ce sujet car, dans la même étude, on peut lire aussi qu'Agia Marina «était habitée des Maronites et que les tombes que l'on retrouve autour sont maronites ", $c f$. ibid., p. 15 et 19.

30. Jeffery 1983, p. 204. 


\section{La Pallourkotissa}

Cette petite chapelle rurale est dédiée à la Vierge Pallourkotissa ${ }^{31}$. Elle est située près d'une conduite d'eau forcée ${ }^{32}$ et présente une seule nef voûtée en berceau, terminée à l'est par une abside semi-circulaire (Fig. 5).

L'étude anthropologique rapporte que cette église était une église franque ${ }^{33}$. Néanmoins, l'aspect architectural de cette construction, dont la nef fut réalisée au moyen de blocs irréguliers, trahit une exécution régionale ouverte aux influences francobyzantines qui caractérise les églises plus vastes édifiées au XVIII ${ }^{\mathrm{e}}$ siècle dans la région, telles celle de Timios Stavros à Potamia, celle dédiée aux saints Andronikos et Athanasia à Dali (datée du début du XIXe siècle par G. Jeffery) ${ }^{34}$. Ces mêmes caractéristiques architecturales se retrouvent aussi dans d'autres églises byzantines chypriotes, telles que Saint-Andronic à Skouli et la Chryseleousa à Polis, au nord de Paphos 35 .

\section{Sainte-Catherine}

\section{Potamia}

Sainte-Catherine ou Santa Caterina, comme l'appellent encore les habitants de Potamia, est une chapelle en ruine dont le vocable est relatif à la Vénitienne Catherine Cornaro, veuve de Jacques II (1460-1473), le dernier des Lusignan à régner sur Chypre. Comme le suggère $\mathrm{C}$. Enlart cette construction, sans doute antérieure au XV $\mathrm{V}^{\mathrm{e}}$ siècle, est à rattacher à la chapelle privée du manoir de Potamia édifié dès le XIV ${ }^{\mathrm{e}}$ siècle.

Seules subsistent aujourd'hui les fondations de cette chapelle. L'étude archéologique de ses vestiges a permis d'identifier deux phases de construction : il est probable qu'à une nef unique se terminant par une abside saillante à l'est, ait succédé un bâtiment comprenant deux nefs achevées à l'est par autant d'absides semi-circulaires ${ }^{36}$.

\section{Saint-Archange}

Cette chapelle totalement ruinée, située près du Yialias, a fait l'objet de sondages archéologiques menés sous la direction de D. Michaelidis au cours du mois de juillet 2000. Seul le mur de fondation ouest de cet édifice affleure au niveau du sol actuel. Il

31. "Pallourkotissa" est un de nombreux vocables de la Vierge signalés à Chypre, $c f$. Delehaye 1907, p. 265-266.

32. L'étude anthropologique indique que non loin de l'église, «il y avait une conduite d'eau forcée qui arrivait à Margon, dans le village de Piroi », cf. Karatsioli 2001, p. 28. D'après un autre passage de cette étude (p. 35), on peut déduire que la même conduite forcée passait juste à côté de Hagia Mavri.

33. Karatsioli 2001, p. 28.

34. Jeffery 1983, p. 202.

35. Curčić 1999, p. 79, fig. 19-20.

36. Voir Lécuyer et al. 2001, p. 670-672, fig. 9. 
était percé d'une ouverture. La largeur de la nef principale est de 5,50 m, tandis qu'un vaisseau latéral semble avoir été ajouté au sud. Le matériel céramique mis au jour date du XVI ${ }^{\mathrm{e}}$ siècle alors que le niveau d'occupation semble être du XIVe siècle. Par ses dimensions, sa technique de construction et l'adjonction d'une nef latérale, cette chapelle se révèle très proche de celle dédiée à sainte Catherine ${ }^{37}$.

\section{Sainte-Croix ou Timios Stavros}

Comme en témoigne une inscription gravée dans le quadrant inférieur d'une plaque ornée d'une croix prophylactique, encastrée à la partie supérieure de la paroi nord de l'église, celle-ci fut achevée et consacrée en 1770. Il s'agit d'un édifice à nef unique voûté d'arêtes (Fig. 6), construit en pierres soigneusement taillées et qui se termine par une abside, polygonale à l'extérieur et semi-circulaire à l'intérieur. Cette particularité de l'abside se retrouve aussi à Saint-Mamas ${ }^{38}$ de Dali, de même que le parement intérieur de l'abside en pierres apparentes, soigneusement taillées et limitées à la partie supérieure par une corniche moulurée. Le type de console à sept denticules, soutenant la retombée des arcs doubleaux des voûtes de la nef, dérive également du modèle des consoles de l'église Saint-Mamas de Dali.

\section{Saint-Démétrianos}

\section{Agridi-Dali}

L'église Saint-Démétrianos est ainsi identifiée grâce à la représentation peinte de ce saint située dans l'arcade aveugle sud-ouest (Fig. 7), et grâce aussi à l'inscription grecque dédicatoire située sur le mur ouest, au-dessus de l'entrée principale. Cette dernière mentionne "l'église... de notre père parmi les saints, Démétrianos Andridiotis... ». L'image du saint est précieuse car elle nous montre Démétrianos vêtu du costume militaire, et indique par là que ce saint ne peut être identifié avec le célèbre évêque homonyme de Chytri-Kythrea ${ }^{39}$, souvent représenté dans les programmes iconographiques des églises chypriotes. D'autres saints homonymes ont été vénérés à Chypre, tel, notamment, Démétrianos martyr de Salamine (III -IVe siècles) ${ }^{40}$ ou Démétrianos de Kophinou. Ce dernier est mentionné dans la chronique de Léontios Machairas avec cinq de ses compagnons martyrs comme faisant partie des trois cents saints allemands issus de Terre-Sainte ${ }^{41}$. L'identification du saint peint dans l'église de Dali est donc

37. Ibid., p. 672-674, fig. 10.

38. Voir infra.

39. D'après sa Vie écrite peu d'années après sa mort, au tout début du $X^{\mathfrak{c}}$ siècle, ce saint se serait illustré dans le cadre des invasions arabes de l'île et aurait obtenu du Calife de Bagdad, auprès duquel il s'était rendu, la liberté de ses diocésains, $c f$. Delchaye 1907, p. 249.

40. Kyrris 1993, p. 214, 222, 224-225; Makarios III 1968, p. 18.

41. Dawkins 1932, vol. 1, §32, 30-33. Kophinou n'est situé qu'à une vingtaine de kilomètres au sud de Dali. Néanmoins, la proximité entre ces deux lieux et la popularité de ce 
malaisée : il s'agit soit du saint martyr de Salamine, soit du saint «allemand » de Kophinou, ou alors - et plus vraisemblablement - d'un personnage résultant d'une fusion de plusieurs traditions hagiographiques. De fait, le calendrier liturgique chypriote, outre le Démétrianos évêque de Chytri, célèbre un second Démétrianos le 23 juin 42.

L'église (Fig. 8) se compose d'une nef unique surmontée d'une coupole soutenue par quatre arcs brisés. L'agencement et l'étagement des volumes décrivent une croix au niveau du toit. Entre ceux-ci, des pendentifs soutiennent le tambour circulaire de la coupole qui est percée de quatre fenêtres étroites. Néanmoins, les bras ouest et est de cette croix sont plus longs que les bras latéraux et accentuent la forme longitudinale du bâtiment. À Chypre, on rencontre dès la fin du $\mathrm{XI}^{\mathrm{e}}$ siècle ce type de construction à divers endroits de l'île. En témoignent les églises dont le riche décor peint atteste des liens évidents avec Constantinople, comme, par exemple, le parecclésion d'Hagia Trias au monastère Saint-Jean-Chrysostome à Koutsouvendis, au nord de Nicosie, la Panagia tou Arakou ou Arakiotissa à Lagoudera, dans le massif du Troodos ${ }^{43}$, de même que des églises beaucoup plus modestes d'époque franque, telle celle de la Panagia Chorteni près de Pélathoussa dans la région de Paphos. Signalons cependant la réelle densité de ce type d'églises au sud de Nicosie. À titre d'exemple, la toute proche église Saint-Georges de Dali (Fig. 9), plus petite que celle de Saint-Démétrianos, présente le même plan. L'église des Saints-Apôtres à Pérachorio, dont les fresques sont datées vers 1180, est également construite suivant un plan similaire; mais sa coupole, de forme irrégulière, repose directement sur les pendentifs et ne comporte pas de tambour ${ }^{44}$.

Le décor peint de Saint-Démétrianos est encore relativement bien conservé. Audessus de la porte principale qui s'ouvre dans la paroi ouest, une inscription dédicatoire fournit la datation de l'achèvement des restaurations et du décor peint, ainsi que le nom des donateurs de l'église : "Michel, fils de Katzouroubis, sa femme et ses enfants ont fait restaurer et peindre cette église en $1317 » 45$. Cette date nous fournit un terminus ante quem pour la réalisation de la construction, et suggère que cette église a été restaurée.

groupe de cinq martyrs thaumaturges ne sont pas des arguments suffisants permettant de valider l'identification de Démétrianos représenté dans l'église de Dali avec le saint homonyme de Kophinou.

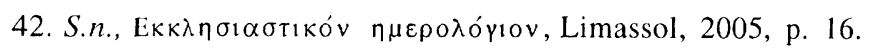

43. Voir à ce sujet Nicolaidès 1996, p. 9 et notes 54, 55 (bibliographie antérieure), et Curčić 1999, p. 77-78, fig. 15-16.

44. Hein, Jakovljević, Kleidt 1998, p. 135-136, fig. 138 ; Stylianou 1985, p. 425-426; surtout Megaw, Hawkins 1962, p. 280-284;

45. Stylianou 1985, p. 425-427. 


\section{Saint-Mamas}

L'église de Saint-Mamas (actuellement cimétériale) située le long du Yalias, à la limite du territoire de l'ancien Idalion, est datée du XVe siècle par C. Enlart ${ }^{46}$ et du $\mathrm{XVI}^{\mathrm{e}}$ siècle par G. Jeffery ${ }^{47}$. Selon $\mathrm{C}$. Enlart, cette église à nef unique de style tardogothique (Fig. 10) aurait été bâtie ou reconstruite après l'invasion mamelouke de Potamia en 1425. La date de 1887 gravée sur la traverse d'imposte de la porte ouest de l'église doit correspondre, tout au moins, à la réfection de l'ensemble des portes en bois de l'édifice.

Saint-Mamas présente un plan rectangulaire terminé à l'est par une abside à pans coupés à l'extérieur, percée d'une petite fenêtre en plein cintre et surmontée d'un oculus. Semi-circulaire à l'intérieur, l'abside présente au sommet de son mur une corniche épanneléc. La nef est partagée en deux travées de voûtes d'arêtes. Deux colonnes engagées soutiennent un arc doubleau qui divise la nef en deux travées. Deux étages d'encorbellements sculptés surmontent les chapiteaux et permettent de soutenir l'arc doubleau.

\section{L'Evangelistria}

Il s'agit d'une vaste église à cinq coupoles, construite en 1930, qui présente un intéressant mélange de styles néo-byzantin et néo-gothique. La totalité de cette église a été construite en pierres de taille ${ }^{48}$. Par ailleurs, le fait qu'une attention particulière ait été accordée au parement des murs, tant extérieur qu'intérieur, doit être mis en relation avec l'influence des édifices de style gothique de la région, à savoir les deux églises dédiées à Saint-Mamas. De même, la forme allongée des fenêtres, les ouvertures en forme de meurtrière et le soubassement mouluré de cette église évoquent, eux aussi, l'architecture gothique de l'île de Chypre.

\section{Conclusions}

L'étude de la topographie cultuelle chrétienne de la région de Potamia-Agios Sozoménos révèle de nombreux saints dédicataires repérés sur un territoire limité : outre la Vierge, on trouve Sozomène, Michel, Mamas, Démétrianos, Marina, Mavri, ou encore Andronikos et Athanasia et Georges. Ces lieux de culte, par leur implantation sur le site étudié, sont à l'évidence en connexion avec l'exploitation agricole du terroir. Plusieurs d'entre eux, tels que l'ermitage Saint-Sozomène, la chapelle de l'Archange,

46. Enlart 1899, p. 199-201.

47. Jeffery 1983, p. 202.

48. Plusieurs témoignages recueillis dans l'étude anthropologique rapportent que les habitants de Dali ont récupéré, pour édifier cette église, des blocs issus des ruines de SaintMamas dans le village d'Agios Sozoménos, cf. Karatsioli 2001, p. 11 et 28. 
Saint-Démétrianos, la Pallourkotissa et Agia Mavri, semblent en effet directement liés à l'apport d'eau, essentiel aux cultures de la région. Par ailleurs, le recours aux reliques de saint Sozomène, au XIVe siècle, pour conjurer certaines calamités - invasions de sauterelles et sécheresse - montre aussi que l'invocation de Sozomène est associée aux préoccupations concrètes des agriculteurs, et il en va de même pour Mamas, le protecteur des bergers.

L'étude de la topographie cultuelle nous apprend enfin que le XIVe siècle constitue une période particulièrement faste pour la région. C'est en effet au cours de ce siècle que le culte de deux saints byzantins locaux, Sozomène et Démétrianos, connaît manifestement un certain succès. En témoignent la deuxième couche de fresques décorant l'ermitage de saint Sozomène et les fresques de l'église dédiée à saint Démétrianos. Les $\mathrm{XV}^{\mathrm{e}}$ et $\mathrm{XVI}^{\mathrm{e}}$ siècles voient aussi l'implantation des deux églises gothiques dédiées à Saint-Mamas, dont les caractéristiques architecturales se retrouvent dans les édifices ultérieurs construits dans les villages modernes de Potamia et de Dali.

Université de Provence, LAMM, Aix-en-Provence Université libre de Bruxelles, Université Marc-Bloch, Strasbourg

Figures 1: LAMM ; 2 : relevé M.-L. Laharie ; 3-10: photos C. Vanderheyde. 


\section{BIBLIOGRAPHIE}

ĆuRěló (S.), 1999, "Byzantine Architecture on Cyprus: An Introduction to the Problem of the Genesis of a Regional Style », dans N. Sevčenko, C. Moss (éd), Medieval Cyprus. Studies in Art, Architecture and History in Memory of Doula Mouriki, Princeton, p. 71-80.

Dawkins (R. M.), 1932, Leontios Makhairas. Recital Concerning the Sweet Land of Cyprus Entitled 'Chronicle', Oxford.

Delehaye (H.), 1907, "Saints de Chypre ", Analecta Bollandiana 26, p. 161301 .

ENLART (C.), 1899, L'art gothique et la Renaissance en Chypre, Paris.

François (V.), Vallauri (L.), 2001, "Production et consommation de céramiques à Potamia (Chypre) de l'époque franque à l'époque ottomane », $B C H \quad 125$, p. 524-546.

Grivaud (G.), 1998, Villages désertés à

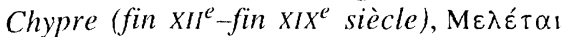

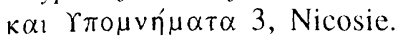

G UNNIS (R.), 1973, Historic Cyprus, Nicosie.

Hein (E.), Jakovljević (A.), Kleidt (B.), 1998, Cyprus. Byzantine Churches and Monasteries. Mosaics and Frescoes, Ratingen.

JEFFERY (G.), 1983, A Description of the Historic Monuments of Cyprus (1 ${ }^{\text {re }}$ éd. 1918), Londres.

Karatsioli (B.), 2001, Potamia et Haghios Sozoménos : mémoire de leurs habitants.

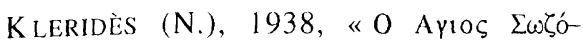

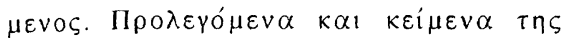

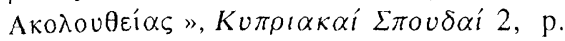
105-110.
KYPRIANOS (Archimandrite), 1902, Iotopía

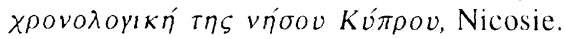

KYRRIS (C.P.), 1993, "The 'Three Hundred Alaman Saints' of Cyprus : Problems of Origin and Identity. A Summary ", dans The Sweet Land of Cyprus, Nicosie, p. 203-235.

KYRRIS (C.P.), 1999, Enciclopedia dei santi. Le chiese orientali, Rome.

LÉCUyer (N.) et alii, 2001, "PotamiaAgios Sozomenos (Chypre). La constitution des paysages dans l'Orient médiéval », $B C H$ 125, p. 655-678.

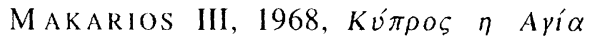
vॅ́oos, Athènes.

Megaw (A. H. S.), Hawkins (J. W.), 1962, "The Church of the Holy Apostles", Dumbarton Oaks Papers 16, p. 277-348.

Mouriki (D.), 1993, "The Cult of Cypriot Saints in Medieval Cyprus as attested by Church Decoration and Icon Painting", dans The Sweet Land of Cyprus, Nicosie, p. 249-251.

Nicolaidès (A.), 1996, "L'église de la Panagia Arakiotissa à Lagoudéra, Chypre: étude iconographique des fresques de $1192 »$, Dumbarton Oaks Papers 50, p. 1-137.

PAPAGEORgIOU (A.), 1999, « $\Lambda \alpha \xi \varepsilon \cup \tau \alpha^{\prime}$

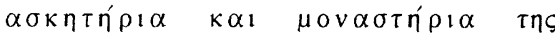

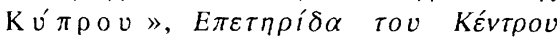

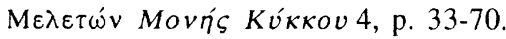

Stylianou (A), Stylianou (J.A.), 1985, The Painted Churches of Cyprus, Treasures of Byzantine Art, Londres. 


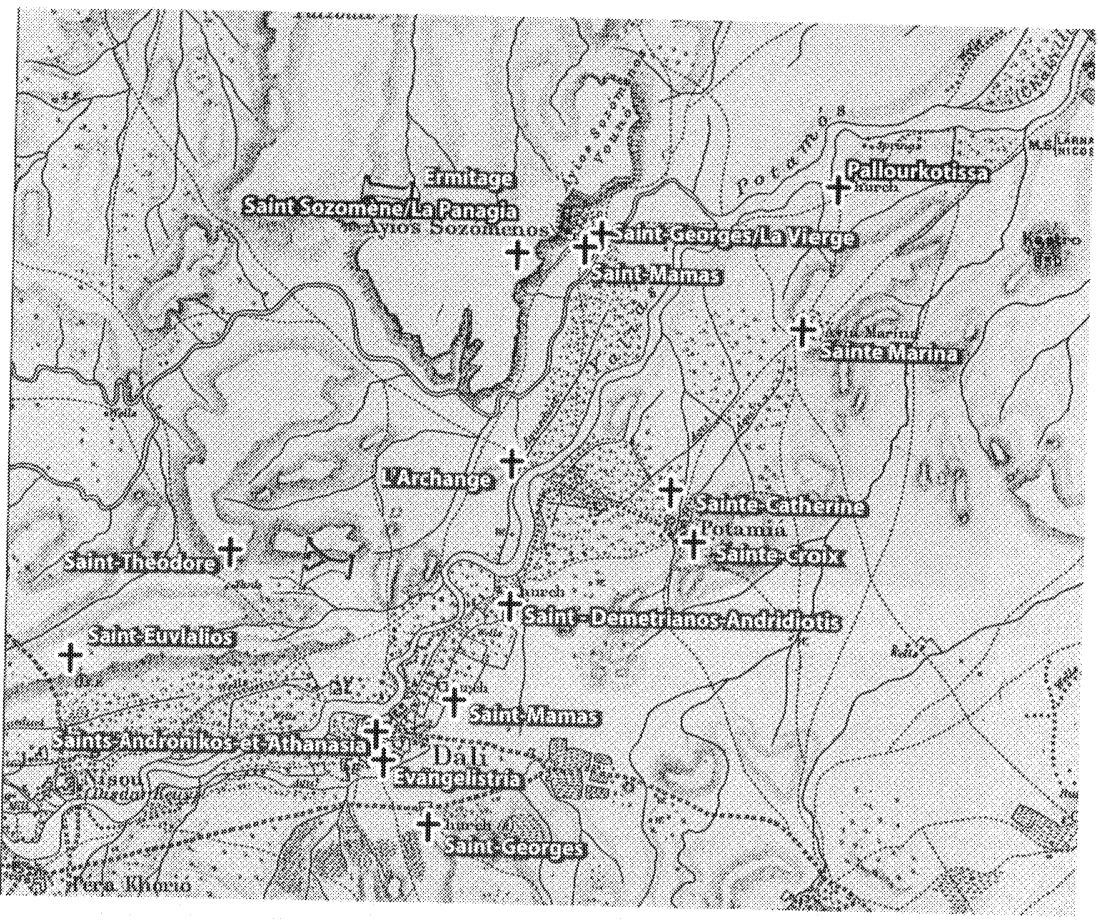

Figure 1. Carte de la région de Potamia-Agios Sozoménos.

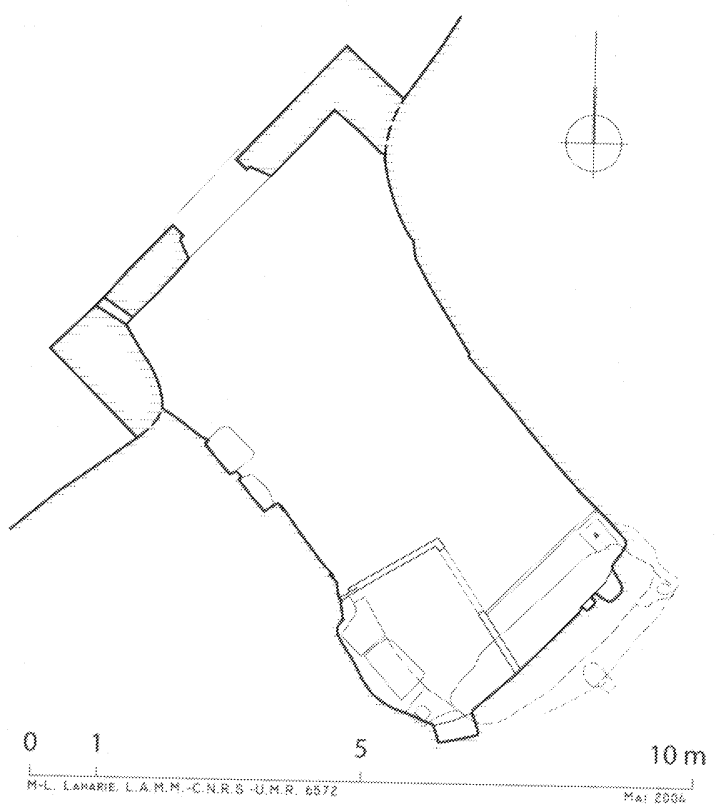

Figure 2. L'ermitage de saint Sozomène (plan de l'état actuel). 


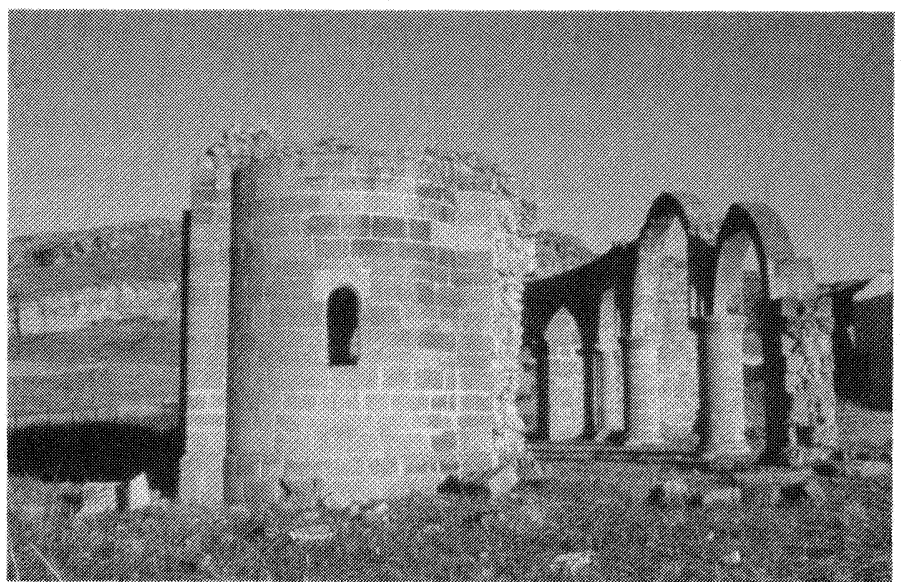

Figure 3. Sain-Mamas du village d'Agios Sozoménos (vue du chevet).

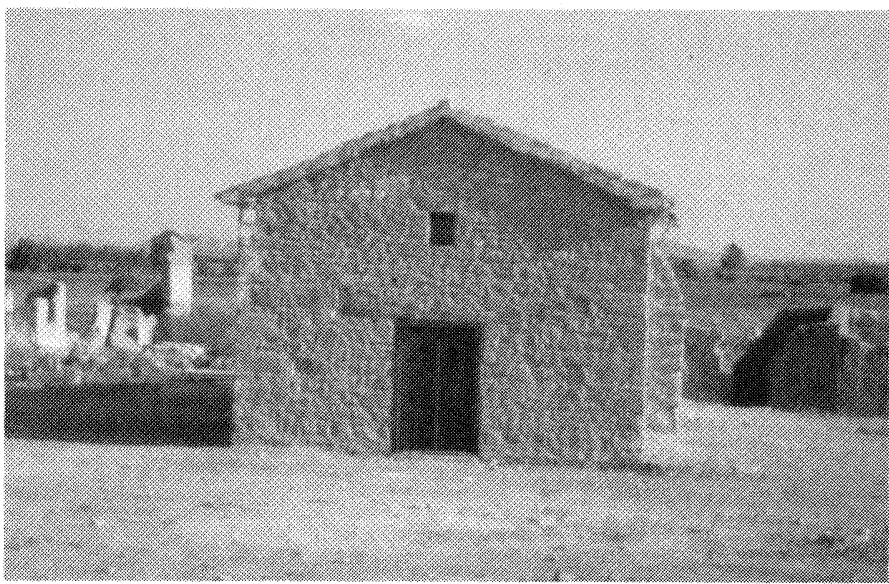

Figure 4. Saint-Georges / la Panagia du village d:Agios Sozoménos.

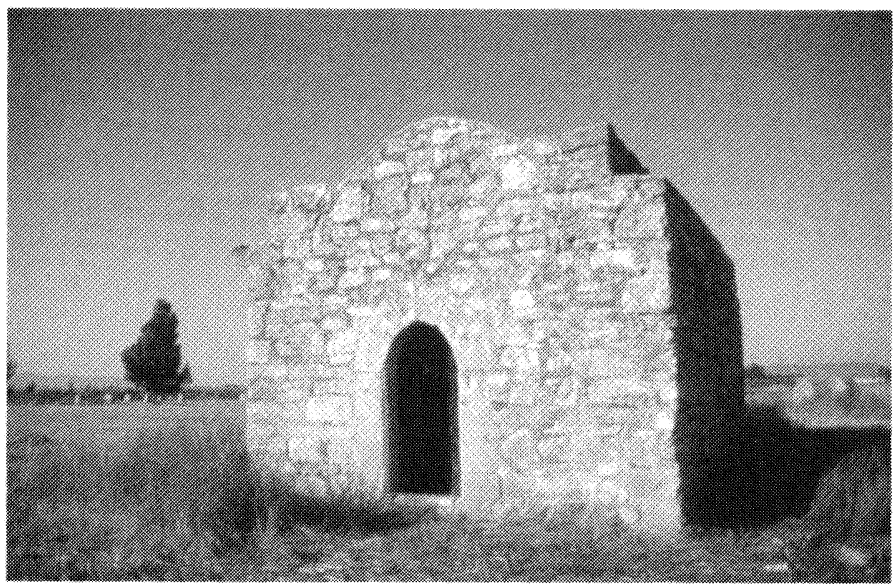

Figure 5. L'eglise de la Vierge Pallowkotissa. 


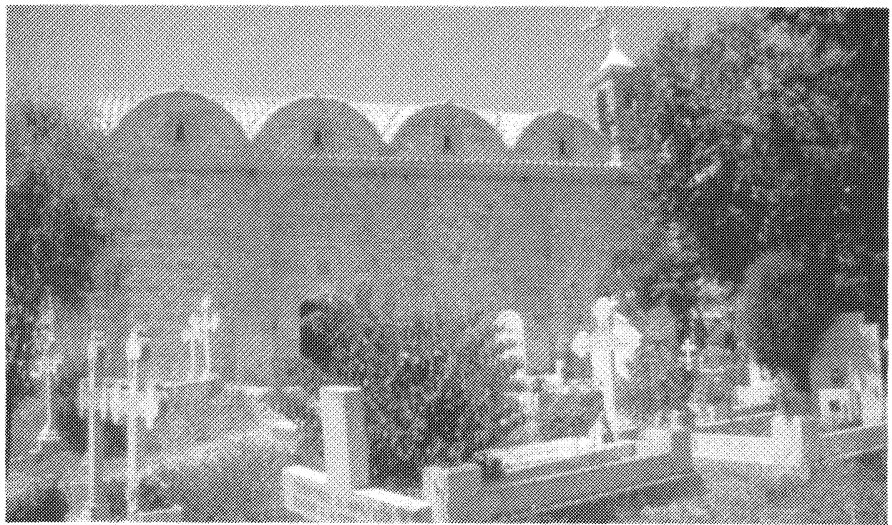

Finne 6. Saine-Croix (facade nond).

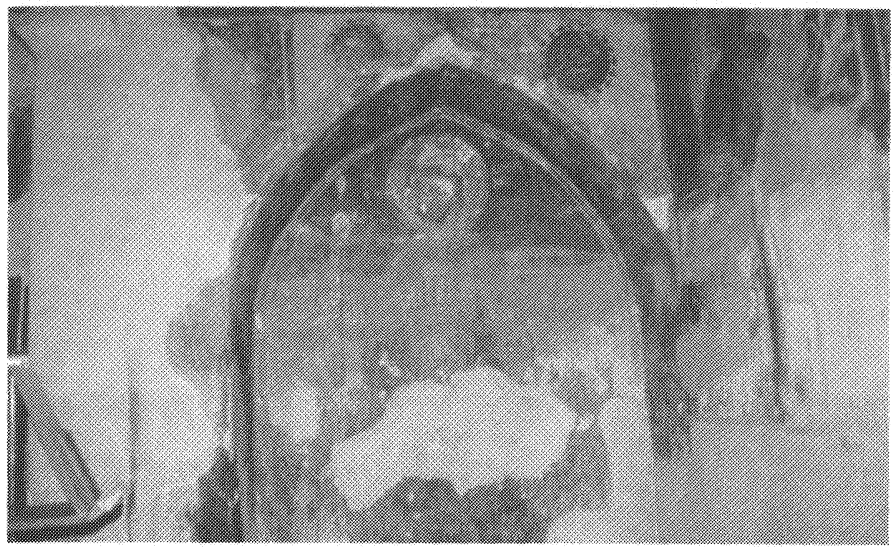

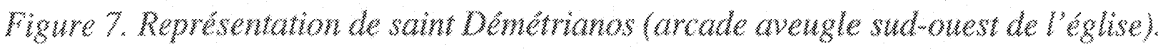

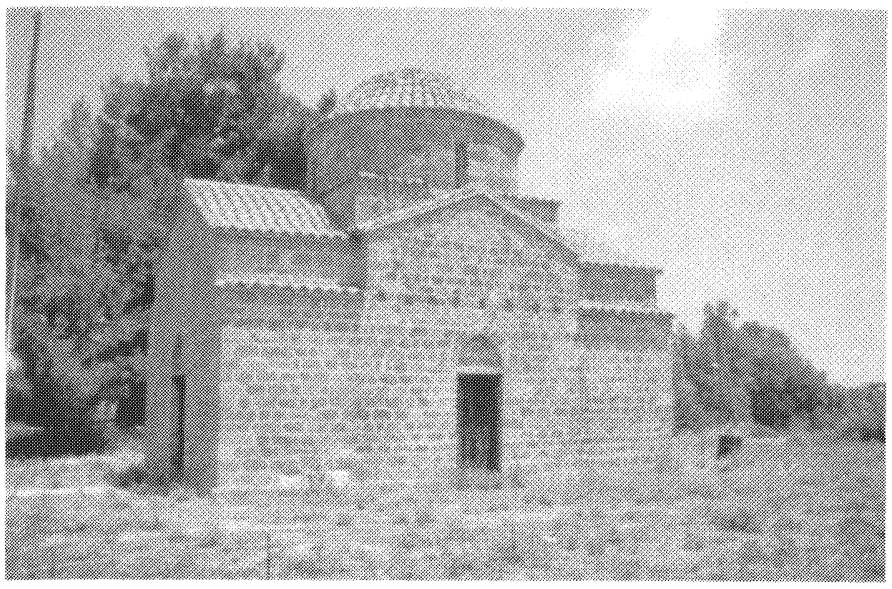

Figure 8. Saim-Dênétrianos (Gagade sud). 


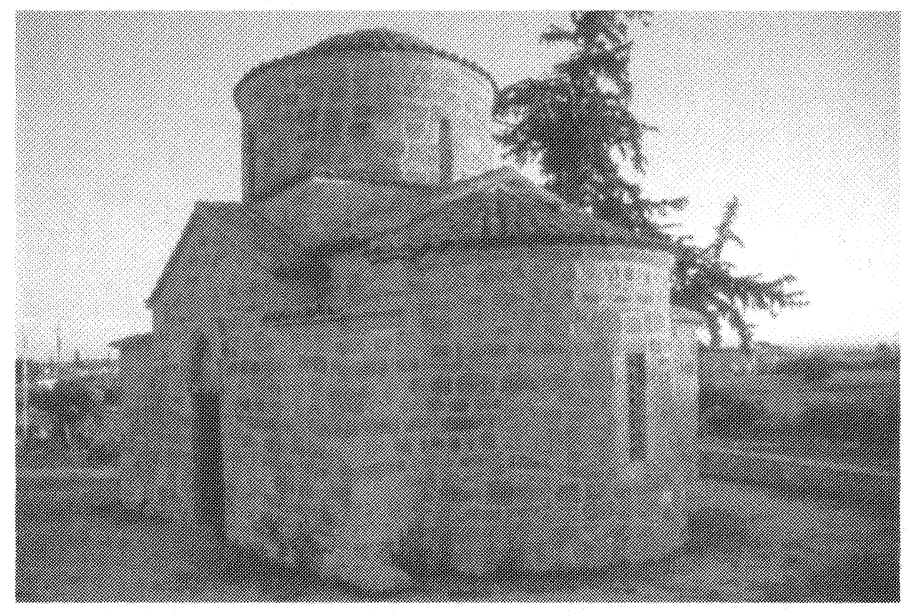

Figure 9. Samt Ceorges do Dal.

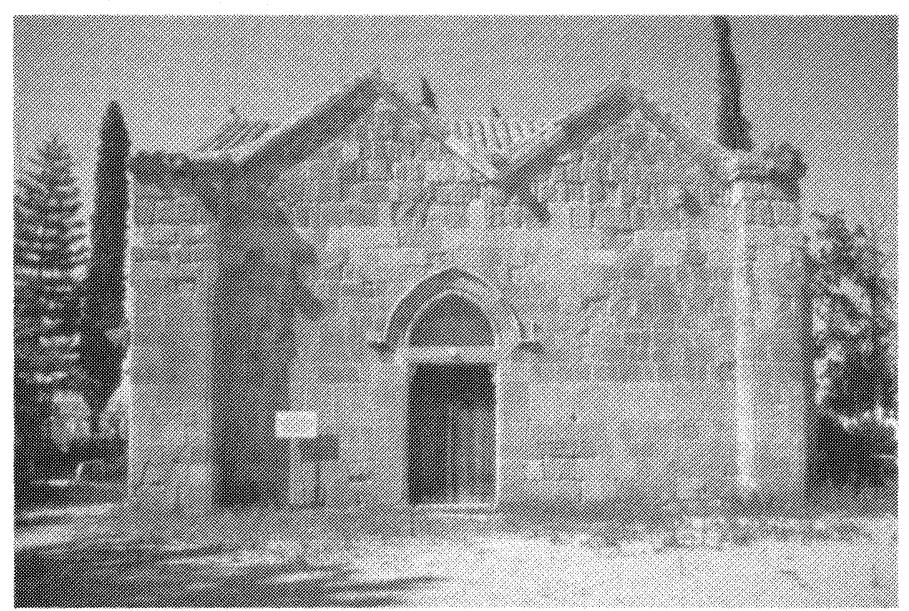

Figure 10. Saint-Mamas à Dali (façade sud). 